\title{
Advantages in diagnosis of giant cell arteritis by ultrasound
}

\author{
Joanna Wojczal', Grzegorz Kozera², Piotr Luchowski', Jolanta Neubauer-Geryk ${ }^{3}$ \\ ${ }^{1}$ Department of Neurology, Ultrasound Lab, Medical University of Lublin, Independent Public University Hospital, Lublin, Poland \\ 2Department of Neurology, Collegium Medicum in Bydgoszcz, Nicolaus Copernicus University in Torun, Poland \\ ${ }^{3}$ Clinical Physiology Unit, Medical Simulation Centre, Medical University of Gdansk, Gdansk, Poland
}

Adv Dermatol Allergol 2019; XXXVI (1): 25-28

DOI: https://doi.org/10.5114/ada.2019.82823

\begin{abstract}
Giant cell arteritis is the most common systemic vasculitis. It selectively affects large- and medium-sized arteries. Arterial wall inflammation leads to luminal occlusion and tissue ischemia, which cause the clinical manifestations of this vasculitis. Susceptible sites include the vascular bed of the cranial nerves and scalp including the temporal arteries, optic nerve, masseter muscles, and the posterior circulation of the central nervous system. Involvement can extend to the aorta and its primary and secondary branches, including the subclavian and axillary arteries, which leads to upper-extremity ischemia. The article presents a review of epidemiology, clinical presentation and especially ultrasound diagnostics of giant cell arteritis.
\end{abstract}

Key words: giant cell arteritis, temporal arteritis, ultrasound, fast track clinic.

\section{Introduction}

Giant cell arteritis (GCA) is the most common systemic vasculitis. It selectively affects large- and mediumsized arteries. Arterial wall inflammation leads to luminal occlusion and tissue ischemia, which cause the clinical manifestations of this vasculitis. Susceptible sites include the vascular bed of the cranial nerves and scalp including the temporal arteries, optic nerve, masseter muscles, and the posterior circulation of the central nervous system. Involvement can extend to the aorta and its primary and secondary branches, including the subclavian and axillary arteries, which leads to upper-extremity ischemia [1].

\section{Epidemiology}

The incidence of GCA among individuals older than 50 years of age varies across geographic regions, being the highest in Scandinavian populations (15-35/100,000 in Sweden [2] and 29.1-32.8/100,000 in southern Norway [3]) and relatively lower in Southern European populations ( 10 per 100,000) [4], and it is infrequent among people of Africa, Asia, Iberia/Iberian Peninsula, and Arabian Peninsula [5]. The male-to-female ratio was $2: 5$ in most studies. The risk increases with advancing age, being 20 times higher after 90 years of age than between
50 and 60 years of age [1]. The age specific incidence rates per 100,000 population increase from 2 in the age group of 50-59 years, to 52 in the age group of 80 and older [6].

A few studies suggest that the incidence of GCA may be rising, even after correction for the aging of the population. Nevertheless, the increases in incidence seem small and consistent with improved knowledge of the disease among physicians [1].

\section{Clinical presentation}

Giant cell arteritis is a heterogeneous disease with a wide range of clinical manifestations, although none of them is pathognomonic because many arteries throughout the body may be affected and produce symptoms and signs that are common to other medical and surgical conditions including dermatologic ones. The signs and symptoms of GCA can be classified into four subsets: manifestations of cranial arteritis, extracranial arteritis, systemic symptoms and polymyalgia rheumatica (PMR) [7]. Patients may develop any combination of these manifestations. Among those, tender, prominent temporal arteries with absent pulses, jaw claudication and diplopia have the highest positive likelihood ratios for GCA diagnosis [8].

Address for correspondence: Joanna Wojczal MD, PhD, Department of Neurology, Medical University of Lublin, Independent Public University Hospital, 8 Jaczewskiego St, 20-954 Lublin, Poland, phone: +48 8172447 20, fax: +48 81724 45 40, e-mail: jwojczal@poczta.onet.pl Received: 20.10.2017, accepted: 13.12.2017. 
Ischemic manifestations include new-onset headaches, scalp tenderness, jaw and upper limb claudication, stroke (particularly of the posterior circulation), visual disturbance, and/or permanent vision loss. Jaw claudication is present in about $50 \%$ of patients and is the clinical feature most highly associated with positive temporal artery biopsy (TAB). Headaches are present in $>60 \%$ of patients, new-onset ones, and are typically, but not always, temporal in position as they may also be frontal, occipital, or generalized. Vision loss, one of the most serious complications of GCA, occurs in up to $15-30 \%$ of cases. An initial visual manifestation of GCA is often amaurosis fugax. This may be caused by anterior optic ischemic neuropathy (AOIN), central or branched retinal occlusion, or choroid infarction, with the first one being the most common cause of blindness in GCA. In GCA patients with monocular blindness, loss of vision in the unaffected eye occurs in $25-50 \%$ of untreated patients. Blindness is rarely reversible even with high-dose glucocorticoids [9].

Scalp necrosis is a rare complication of GCA. It is considered to be a result of occlusion of the majority of the 4 arteries supplying the temporal region of the scalp (arteriae temporales/temporal arteries, arteriae frontales/ frontal arteries, arteriae retroauriculares/retroauricular arteries, arteriae occipitales/occipital arteries). The probability of necrosis likely depends on the degree of anastomoses between these vessels. It is likely that the number of obstructed vessels increases with the duration of disease. A correlation between delay of diagnosis (and start of appropriate treatment) and onset of scalp necrosis is supported by the fact that there is no single case in the literature in which scalp necrosis occurred after adequate treatment had been started [10].

Involvement of branches of the aortic arch (particularly the subclavian and axillary arteries) can cause arm

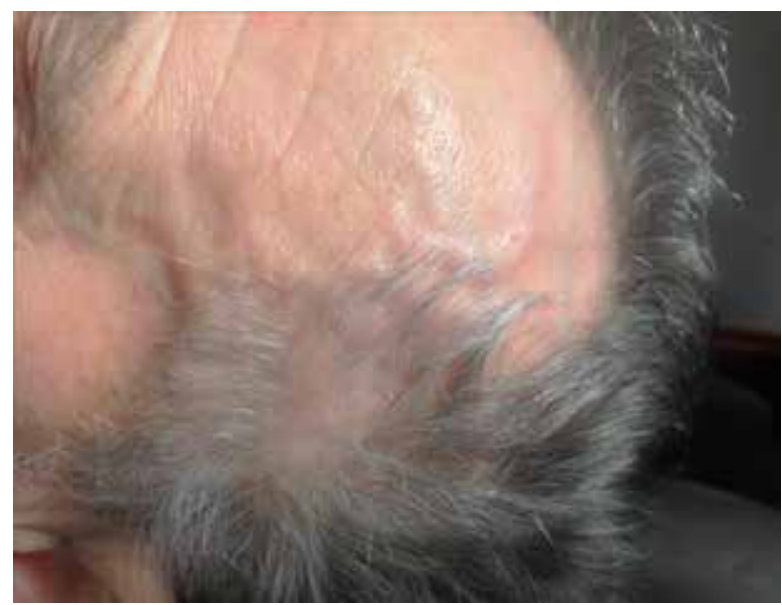

Figure 1. Prominent temporal artery and its branches accompanied by local redness and skin edema in a patient with GCA claudication. Several studies comparing GCA patients with large vessel disease (axillary/subclavian involvement demonstrated in angiography) to those with cranial involvement, showed differences in important clinical aspects. The GCA patients with large-vessel involvement were likely to be younger, less likely to present with headaches, less likely to have a positive TAB, at a lower risk of vision loss, required a higher glucocorticoid dose, and at a higher risk of relapse [11].

Temporal artery abnormalities such as prominent or enlarged temporal artery, absent temporal artery pulse, or temporal artery tenderness accompanied by local redness and skin edema (Figure 1) may be revealed by physical examination. Arterial pulses may be absent or asymmetric in the presence of large-vessel involvement. In addition, bruits in carotid or subclavicular areas may be present, as well as asymmetric arm blood pressure readings. Vascular physical examination in GCA has recently been shown to have low sensitivity but high specificity in detecting the disease demonstrated in angiography [12].

Systemic features are frequent and include low-grade fever (50\%), fatigue, anorexia, and weight loss. Fever may exceed $39^{\circ} \mathrm{C}$ in up to $15 \%$ of patients. The GCA may present as fever of unknown origin (FUO), and may account for up to $16 \%$ of cases of FUO in the elderly [13]. Up to $50 \%$ of patients present with symptoms of polymyalgia rheumatica (PMR), including early morning stiffness and pain in the shoulder and/or hip girdle [9].

\section{Diagnostics}

For the purpose of differentiating GCA from other forms of vasculitis, the American College of Rheumatology formulated five classification criteria for GCA: age $\geq 50$ years at onset, localized headache of new onset, tenderness or decreased pulse of the temporal artery, erythrocyte sedimentation rate $>50 \mathrm{~mm} / \mathrm{h}$ and biopsy revealing necrotizing arteritis. The presence of three of these five criteria is associated with $94 \%$ sensitivity and 91\% specificity for the diagnosis of GCA [14].

Temporal artery biopsy has been for a long time the gold standard test for establishing the diagnosis, with a high specificity but low sensitivity. It can be misleading in a significant number of cases. Up to $44 \%$ of patients with clinical features of GCA have a negative biopsy. There are many reasons for this, including the adequacy of the specimen obtained, the duration of glucocorticoid treatment prior to biopsy and the presence of skip lesions (intermittent, dispersed areas of abnormality in the artery, which might be missed because not all areas of the artery will be sectioned for examination). Ultrasound and other imaging techniques are emerging as alternative tests to biopsy but have not been used widely. UItrasound imaging can be used to assess both temporal arteries as well as both axillary arteries, which has been shown to increase the diagnostic yield [15]. 


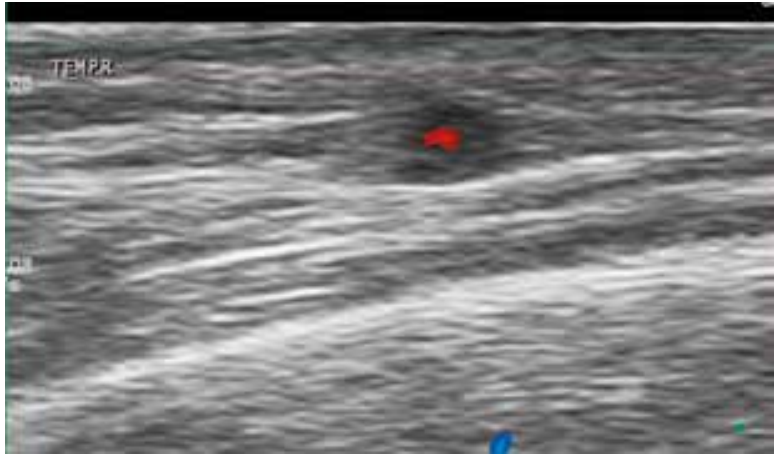

Figure 2. Wall swelling ("halo sign") in cross section colourcoded Doppler ultrasound of the branch of temporal artery diagnostics for acute temporal arteritis. The examination was performed with ultrasound probe $13 \mathrm{MHz}$

In fact, ultrasound has the highest resolution of all the imaging techniques used for the diagnosis of vasculitis. Today, a high-frequency probe of more than $10 \mathrm{MHz}$ (10-22 MHz) provides both an axial and lateral resolution of $0.1 \mathrm{~mm}(100 \mu \mathrm{m})$. Ultrasound depicts the artery wall and provides information about blood-flow characteristics within the artery. Color Doppler ultrasound of temporal arteries shows hypoechoic (dark) edematous wall swelling ("halo sign") in acute temporal arteritis [16] (Figure 2). This edematous tissue is not compressible contrary to lumen of the artery fulfilled by color coded flow [17] (Figure 3). The "halo" disappears with glucocorticoid treatment after $2-3$ weeks in most patients [15], similarly to skin abnormalities.

The diameter of the halo is $0.3-2 \mathrm{~mm}$. Stenosis and occlusion were less specific for GCA than the halo sign [18]. Subsequent studies, however, showed poorer performance of the halo sign, which was not always superior over clinical ACR criteria [19, 20].

There has been significant interest in whether color Doppler could replace TAB as the gold standard for diagnosing GCA [18, 21]. Meta-analysis in 2005 [22] concluded that the hypoechoic halo was highly specific (82\%), but unfortunately not very sensitive, for temporal arteritis (69\%). Subsequent meta-analyses by Arida et al. [23] and Ball et al. [24] found similar values for specificity $(91 \%$ and $83 \%$, respectively) and sensitivity (68\% and $75 \%$, respectively).

With increasing experience and quality of ultrasound equipment, more centers achieve reliable results with ultrasound examination and replace temporal artery biopsy in cases with definitive clinical and ultrasound findings $[15,25]$.

A recent large, prospective multicenter cohort study (TABUL study), comparing TAB to ultrasound of temporal and axillary arteries for diagnosis of newly suspected GCA cases has shown that ultrasound was better than biopsy at identifying patients who did have GCA: it identified $54 \%$ of these patients compared with $39 \%$ identified

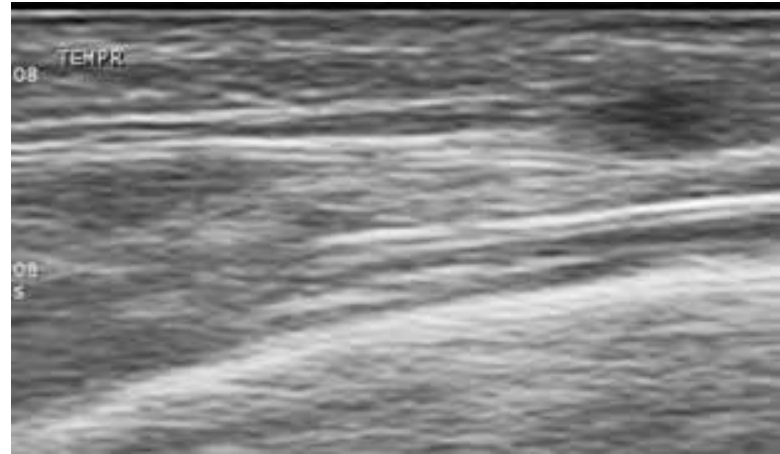

Figure 3. Arterial compression test - the same example as in Figure 2 - after compression with the ultrasound probe, the color flow inside the artery lumen disappears while the arterial wall swelling ("halo") persists

from biopsy, however the specificity was lower (81\% vs. $100 \%)$. Biopsy performed better than ultrasound in the patients who did not have GCA: none of these patients had a positive biopsy, whereas $19 \%$ had a positive scan. A strategy that involves scanning all patients with suspected GCA identified 93\% of those patients with GCA. This strategy was also cheaper than the current practice of relying on a doctor's assessment and biopsy alone [15]. About $50 \%$ of newly diagnosed GCA patients have axillary artery involvement. Including ultrasound examination of the axillary arteries in a protocol increased the diagnostic yield for GCA in routine practice, particularly as only $60 \%$ of patients with large-vessel GCA have temporal artery involvement. An experienced sonographer examining both the temporal and axillary arteries should find more GCA patients than biopsy with histology of 1-2 $\mathrm{cm}$-long segments of one of the branches of the common superficial temporal arteries [16]. Fast-track outpatient clinics for the diagnosis of GCA help initiate treatment before complications such as blindness occur. In such clinics, patients receive appointments within $24 \mathrm{~h}$. Clinical examination and ultrasound of temporal and axillary arteries are performed by an experienced ultrasonographer [26]. Other arteries may be examined depending on clinical findings. In most cases this clearly determines if GCA is present. A recent study included newly diagnosed GCA patients in a fast-track GCA clinic in whom intimamedia thickness (IMT) measurement was performed at or within $24 \mathrm{~h}$ after diagnosis and matched controls. The study found that IMT measurement can correctly distinguish vasculitic from normal arteries in suspected GCA (cut-off values are 0.42, 0.34, 0.29, 0.37 and $1.0 \mathrm{~mm}$, respectively, with $100 \%$ sensitivity and specificity for common superficial temporal arteries, for frontal branches and for axillary arteries and sensitivity of $97.2 \%$ and $87.5 \%$ and specificity of $98.7 \%$ and $98.8 \%$ for parietal branches and facial arteries, respectively) [27].

Temporal artery biopsy is performed in ambivalent cases. Ultrasound may be important in the search for 
the better segment of the artery to perform the biopsy, raising its sensibility [21]. Other imaging techniques such as magnetic resonance imaging (MRI), computed tomography angiography (MRA), computed tomography (CT), magnetic resonance angiography (CTA) or positron emission tomography (PET) can be carried out, particularly if aortitis is suspected and ultrasound findings are not clear. Conventional angiography is only used for interventional treatment. In particular ultrasound is a valuable tool for follow up, especially for large-vessel GCA [16].

\section{Conclusions}

In GCA patients, ultrasound of temporal and axillary arteries has many advantages: it is widely available and it can be performed by the physician himself. The suspected diagnosis can be confirmed or excluded immediately. Ultrasound is not invasive and has no relevant side effects. Findings can be explained to the patient during the examination. The sonographer needs to be well trained in vascular ultrasound in order to provide reliable results. Good reliability is possible as mentioned above. Fast track clinics help establish the diagnosis early and thus may prevent complications such as blindness.

\section{Conflict of interest}

The authors declare no conflict of interest.

\section{References}

1. Chew SSL, Kerr NM, Danesh-Meyer HV. Giant cell arteritis. J Clin Neurosci 2009; 16: 1263-68.

2. Petursdottir V, Johansson H, Nordborg E, et al. The epidemiology of biopsy-positive giant cell arteritis: special reference to cyclic fluctuations. Rheumatology (Oxford) 1999; 38: 1208-12.

3. Haugeberg G, Paulsen PQ, Bie RB. Temporal arteritis in Vest Agder County in southern Norway: incidence and clinical findings. J Rheumatol 2000; 27: 2624-7.

4. Weyand CM, Goronzy JJ. Clinical practice. Giant-cell arteritis and polymyalgia rheumatica. N Engl J Med 2014; 371: 50-7.

5. Borchers AT, Gershwin ME. Giant cell arteritis: a review of classification, pathophysiology, geoepidemiology and treatment. Autoimmun Rev 2012; 11: A544-54.

6. Salvarani C, Crowson CS, O'Fallon WM, et al. Reappraisal of the epidemiology of giant cell arteritis in Olmsted County, Minnesota, over fifty-year period. Arthritis Rheum 2004; 51: 264-8.

7. Nesher G. The diagnosis and classification of giant cell arteritis. J Autoimmun 2014; 48-49: 73-5.

8. Seo P, Stone JH. Large-vessel vasculitis. Arthritis Rheum 2004; 51: 128-39.

9. Ninan J, Lester S, Hill C. Giant cell arteritis. Best Pract Res Clin Rheumatol 2016; 30: 169-88.

10. Tsianakas A, Ehrchen JM, Presser D, et al. Scalp necrosis in giant cell arteritis: case report and review of the relevance of this cutaneous sign of large-vessel vasculitis. J Am Acad Dermatol 2009; 61: 701-6.
11. Muratore F, Kermani TA, Crowson CS, et al. Large-vessel giant cell arteritis: a cohort study. Rheumatology (Oxford) 2015; 54: 463-70.

12. Grayson PC, Tomasson G, Cuthbertson D, et al. Association of vascular physical examination findings and arteriographic lesions in large vessel vasculitis. J Rheumatol 2012; 39: 303-9.

13. Knockaert DC, Vanneste LJ, Bobbaers HJ. Fever of unknown origin in elderly patients. J Am Geriatr Soc 1993; 41: 1187-92.

14. Hunder GG, Bloch DA, Michel BA, et al. The American College of Rheumatology 1990 criteria for the classification of giant cell arteritis. Arthritis Rheum 1990; 33: 1122-8.

15. Luqmani R, Lee $E$, Singh S, et al. The role of ultrasound compared to biopsy of temporal arteries in the diagnosis and treatment of giant cell arteritis (TABUL): a diagnostic accuracy and cost-effectiveness study. Health Technol Assess 2016; 20: 1-238.

16. Schmidt WA. Role of ultrasound in the understanding and management of vasculitis. Ther Adv Musculoskel Dis 2014; 6: 39-47.

17. Aschwanden M, Daikeler T, Kesten F, et al. Temporal artery compression sign - a novel ultrasound finding for the diagnosis of giant cell arteritis. Ultraschall Med 2013; 34: 47-50.

18. Schmidt WA, Kraft H, Vorpahl K, et al. Color duplex ultrasonography in the diagnosis of temporal arteritis. N Engl J Med 1997; 337: 1336-42.

19. Salvarani C, Silingardi M, Ghirarduzzi A, et al. Is duplex ultrasonography useful for the diagnosis of giant-cell arteritis? Ann Intern Med 2002; 137: 232-8.

20. Reinhard M, Schmidt D, Hetzel A. Color-coded sonography in suspected temporal arteritis - experiences after 83 cases. Rheumatol Int 2004; 24: 340-6.

21. Karahaliou M, Vaiopoulos G, Papaspyrou S, et al. Colour duplex sonography of temporal arteries before decision for biopsy: a prospective study in 55 patients with suspected giant cell arteritis. Arthritis Res Ther 2006; 8: R116.

22. Karassa FB, Matsagas MI, Schmidt WA, et al. Metaanalysis: test performance of ultrasonography for giant-cell arteritis. Ann Intern Med 2005; 142: 359-69.

23. Arida A, Kyprianou M, Kanakis M, et al. The diagnostic value of ultrasonography-derived edema of the temporal artery wall in giant cell arteritis: a second meta-analysis. BMC Musculoskelet Disord 2010; 11: 44.

24. Ball EL, Walsh SR, Tang TY, et al. Role of ultrasonography in the diagnosis of temporal arteritis. Br I Surg 2010; 97 : 1765-71.

25. Alberts M. Temporal arteritis: improving patient evaluation with a new protocol. Perm J 2013; 17: 56-62.

26. Diamantopoulos AP, Haugeberg G, Lindland A, et al. The fast-track ultrasound clinic for early diagnosis of giant cell arteritis significantly reduces permanent visual impairment: towards a more effective strategy to improve clinical outcome in giant cell arteritis? Rheumatology (Oxford) 2016; 55: 66-70

27. Schäfer VS, Juche A, Ramiro S, et al. Ultrasound cut-off values for intima-media thickness of temporal, facial and axillary arteries in giant cell arteritis. Rheumatology (Oxford) 2017; 56: 1479-83. 